\title{
Gas flow calibrations performed at the National Metrology Institute of South Africa (NMISA)
}

\author{
D. Jonker^ and E.P. Tarnow \\ National Metrology Institute of South Africa (NMISA), Pretoria, South Africa
}

Received: 24 September 2014 / Accepted: 11 June 2014

\begin{abstract}
The National Metrology Institute of South Africa (NMISA) Gas Flow Laboratory provides traceability to the South African Industry for gas flow measurements. A new primary standard for gas flow calibrations was purchased and commissioned. With three flow cells, a flow range of $0.5 \mathrm{~mL} / \mathrm{min}$ to $50000 \mathrm{~mL} / \mathrm{min}$ is covered. The main features of this standard are accuracy, speed and convenience. This paper describes the activities of the NMISA Gas Flow Laboratory - a discussion of the primary standard, the validation thereof, calibration methods for client instrumentation, analysis of measurement results and the calculation of measurement uncertainties.
\end{abstract}

Keywords: Traceability, gas flow measurements, primary standard, calibration methods, measurement uncertainties

\section{Introduction}

The National Metrology Institute of South Africa (NMISA) is mandated to provide the South African industry with traceable measurements. Gas flow traceability was previously provided through a Califlow MKS primary gas flow standard. The Califlow MKS has become obsolete since it is no longer supported by the manufacturer and has become unreliable. Therefore a new primary standard for gas flow measurement has been purchased to replace the Califlow MKS instrument [1].

The NMISA gas flow laboratory calibrates a wide variety of flow instrumentation for the industry, especially instruments used for environmental monitoring.

\section{The NMISA gas flow laboratory}

The gas flow laboratory is part of the Flow Section in the Physical Metrology Division. The Flow Section consists of the Pressure, Viscosity and Gas Flow laboratories. The laboratory is staffed by one metrologist who is responsible for all gas flow calibrations. Currently, only gas flow calibration services are offered. The flow range covered is from $0.5 \mathrm{~mL} / \mathrm{min}$ to $50000 \mathrm{~mL} / \mathrm{min}$. Nitrogen gas is used as the flow medium. Typical instruments received for calibration include mass flow controllers, mass flow meters, bubble flow meters and rotameters.

The gas flow laboratory is equipped with the following equipment:

- Bios Met Lab ML-800 primary flow standard consisting of three flow cells.

^ Correspondence: djonker@nmisa.org
- Bios Integrator 110-Met Lab Command \& Control Module.

- Bios Gas Flow Bench.

- Vane anemometer (currently out of service).

- Bubble flow meter.

- Environmental monitoring equipment (barometers; temperature- and humidity loggers).

The NMISA gas flow laboratory is not yet accredited to ISO/IEC 17025. At the moment measurements are traceable to international standards held at NIST (National Institute of Science and Technology). NMISA is aiming to achieve SANAS accreditation in 2014 and in-house traceability shortly thereafter [1].

\section{NMISA primary standard for gas flow calibrations}

The Bios Met Lab ML-800 was purchased as a primary standard for gas flow calibrations at NMISA. The ML-800 is a positive displacement primary piston prover and can be used for gas flow measurements in pressure or vacuum applications. The ML-800 complies with the requirements necessary for a primary standard, namely that flow measurements are made in terms of volume per unit time. This is accomplished by means of a piston moving in a cylinder of known cross-sectional area over a measured distance in a measured time.

Volumetric or standardised flow readings can be displayed at the push of a button, the standardised values being calculated from the pressure, as measured by the built in barometer in the ML-800 and a reference temperature, as entered by the metrologist. Measurements can 
be performed manually (one at a time), or automatically. Up to 100 measurements can be performed in an averaging sequence. The ML-800 measures gas flow rates at the measured atmospheric pressure $\pm 7 \mathrm{mmHg}$.

The ML-800 consists of two primary components - a common base and selectable flow cells. The main computer and timing crystal are housed in the base component as well as a precision barometer. The various flow cells plug into the base to create a functional system - the two components cannot operate independently.

The flow cells consist of a borosilicate glass tube with a precision-machined piston. In addition, they also contain an integrated temperature sensor and barometric pressure transducer in the gas flow stream for instant conversion of the volumetric readings into standardised flow.

Four interchangeable flow cell models are available:

- ML-800 Ultra Low Flow Cell: ML-800-3 (in use at NMISA)

Flow range: 0.5 to $50 \mathrm{sccm}(\mathrm{mL} / \mathrm{min})$

Accuracy: $\pm 0.25 \%$;

- ML-800 Low Flow Cell: ML-800-10 (future purchase being considered)

Flow range: 5 to $500 \mathrm{sccm}(\mathrm{mL} / \mathrm{min})$

Accuracy: $\pm 0.15 \%$;

- ML-800 Medium Flow Cell: ML-800-24 (in use at NMISA)

Flow range: 50 to $5000 \mathrm{sccm}(\mathrm{mL} / \mathrm{min})$

Accuracy: $\pm 0.15 \%$;

- ML-800 High Flow Cell: ML-800-44 (in use at NMISA) Flow range: 500 to $50000 \mathrm{sccm}(\mathrm{mL} / \mathrm{min})$

Accuracy: $\pm 0.15 \%$.

The measurements performed with the ML- 800 are traceable to international standards. Before dispatch, the standard was calibrated by the calibration laboratory of the supplier, which is NVLAP (National Voluntary Laboratory Accreditation Programme in the USA) accredited. The Clock Period of the timing crystal and the barometer of the base unit were calibrated. The ultra-low flow cell was gravimetrically calibrated together with its temperature and pressure sensors. The medium and high flow cells were dimensionally calibrated together with their temperature and pressure sensors. For each flow cell, temperature and pressure corrections were applied to the volumetric flow readings to obtain standardised flow readings [2].

\section{Validation of the Bios Met Lab ML-800 primary flow standard}

\subsection{Purpose of the validation}

The purpose of the validation measurements was to ensure that the flow standard was not damaged during shipping from the United States of America to South Africa. The validation measurements were also required to provide objective evidence to demonstrate the competence of the new flow metrologist in the operation of the ML-800 and the calibration of flow instrumentation [3].

\subsection{Validation method}

Due to the very limited resources together with severe budgetary constraints, the ML- 800 was used to calibrate two mass flow controllers (S/N 0858300004 \& 0778900001 ) of an API Dynamic Dilution Calibrator (Model: 700; Serial Number: 777). These mass flow controllers had been calibrated against the Califlow MKS primary gas flow standard, Type A200, serial number 95068002N previously used in the NMISA flow laboratory. Nitrogen gas was used as the flow medium.

Whilst this was far from an ideal validation exercise, since the mass flow controllers were of a lower accuracy than the ML-800, it was still considered to be valuable to establish confidence and competence of the metrologist, as well as identifying any significant shifts in the ML-800 performance after transit from the USA to South Africa.

The ML-800 and the API Calibrator, which controlled the mass flow controllers being calibrated, were operated according to the procedures contained in their respective operating manuals.

The two mass flow controllers were calibrated against the ML-800, one at a time. The inlet port of the mass flow controller being calibrated was connected to the nitrogen gas cylinder and the outlet port to the inlet port of the appropriate ML-800 flow cell. Connections to the mass flow controllers were made directly to the inlet and outlet ports and not to the ports at the rear of the calibrator. The reason for this was to ensure there was no gas diffusion/leakage possible via any other gas paths, and that the full gas stream passed through the mass flow controller being calibrated.

Before any measurements were performed, the gas path was investigated for leaks by means of pressurising the system to $200 \mathrm{kPa}$ and then using Snoop liquid leak detector fluid.

Each mass flow controller was calibrated at twenty points and for each calibration point, ten measurements were performed and an average calculated. The measurement results were recorded manually on a worksheet. Ambient conditions were also recorded [3].

\subsection{Validation results}

The "Ref Val" values in the graphs in Figures 1 and 2 were the values as obtained through calibration against the Califlow MKS instrument. The "Lab Val" values were obtained through calibration against the new ML-800 instrument.

The "Lab Val" values have been intentionally offset on the $x$-axis for clarity.

Figure 1 shows that the laboratory values obtained with the ML-800 are higher than the reference values which were measured with the Califlow MKS. The measurement uncertainties calculated for the laboratory values are smaller than that of the reference values. At the nominal flow rates of $2 \mathrm{~L} / \mathrm{min}$ to $8 \mathrm{~L} / \mathrm{min}, 19 \mathrm{~L} / \mathrm{min}$ and 


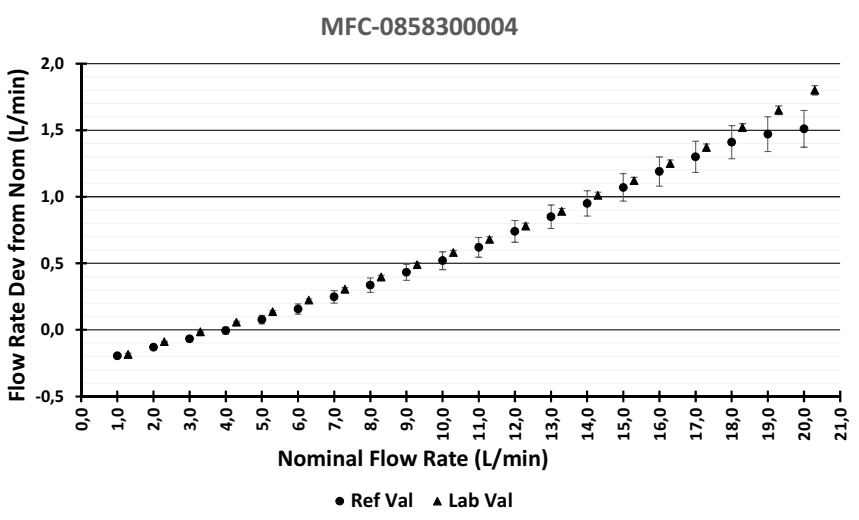

Fig. 1. Flow measurement deviations from nominal flow rate for MFC-0858300004.

$20 \mathrm{~L} / \mathrm{min}$, the measurement uncertainties of the reference and laboratory values are not overlapping. This indicates that the calculated normalised error $\left(E_{n}\right)$ values are greater than one at these points. The measurement results of the two calibrations are therefore not equivalent.

In Figure 2, again the the laboratory values obtained with the ML-800 seemed to be higher than the reference values which were measured with the Califlow MKS, except for the nominal flow rates of $0.01 \mathrm{~L} / \mathrm{min}$ to $0.03 \mathrm{~L} / \mathrm{min}$. At $0.01 \mathrm{~L} / \mathrm{min}$ and $0.02 \mathrm{~L} / \mathrm{min}$ the reference values are higher and at $0.03 \mathrm{~L} / \mathrm{min}$ the two values are exactly the same. At nominal flow rates up to $0.08 \mathrm{~L} / \mathrm{min}$, the measurement uncertainties of the two calibrations are comparable, while at the higher flow rates the uncertainties of the reference values are notably larger. Measurement uncertainties of the reference and laboratory values are not overlapping for most of the nominal flow rates. The calculated normalised error $\left(E_{n}\right)$ values varied between 1.00 and $2.58\left(E_{n}=-0.43\right.$ and 0.00 for flow rates of $0.02 \mathrm{~L} / \mathrm{min}$ and $0.03 \mathrm{~L} / \mathrm{min}$ respectively). Measurement results of the two calibrations are therefore not equivalent.

Possible reasons for the differences in measurement results are:

1) Drift of the mass flow controllers since their calibration against the Califlow MKS;

2) Difference in calibration set-up; slightly different method:

3) The mass flow controllers were calibrated against the Califlow MKS shortly before its removal from service. The calibration accuracy of the the Califlow MKS may have been questionable.

\section{Calibration methods}

Flow instruments are calibrated by connecting them in line with the reference standard. The inlet ports of mass flow controllers are connected to the gas cylinder supplying the flow medium, while the outlet ports supply gas to the ML-800 reference standard. Bubble flow meters are

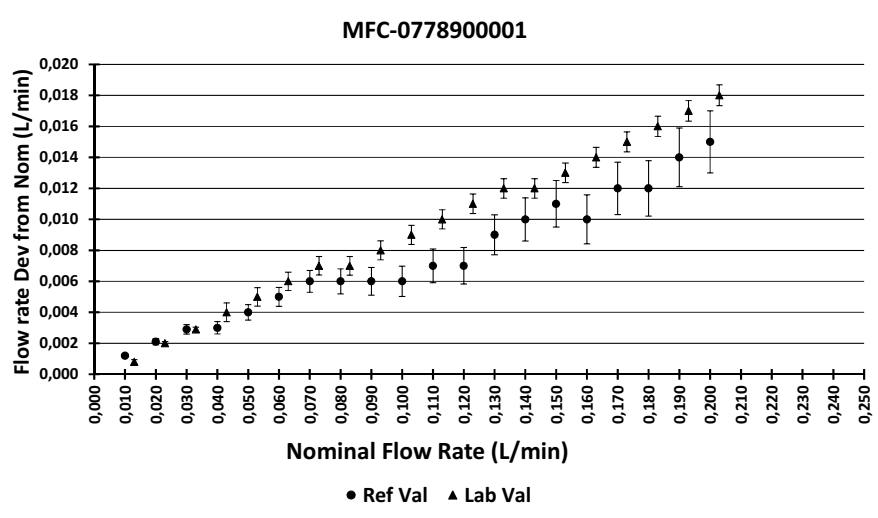

Fig. 2. Flow measurement deviations from nominal flow rate for MFC-0778900001.

connected to the outlet of the reference standard. The reason for this method is to protect the reference standard from possible soap solution contamination of the flow cells.

Furthermore for each calibration performed, the volumetric flow readings are converted to standardised flow readings at standard temperature and pressure as requested by the client, using the following equation:

$$
\text { Volumetric flow }=Q \times\left(T_{m} / T_{s t d}\right) \times\left(P_{s t d} / P_{m}\right)
$$

where

$Q$ is the Standard flow rate;

$T_{m}$ is the measured temperature of the gas in the flow tube;

$T_{s t d}$ is the standard temperature as requested by client; $P_{m}$ is the gas pressure measured in the flow tube; $P_{s t d}$ is the standard pressure as requested by client [1].

\subsection{Effects of unit under test calibration position}

Flow meters are connected in line with the reference standard or to the outlet of the standard, depending on the design of the flow meter. In order to establish whether or not the position of the unit under test (UUT) in the flow line influences the measurement results, measurements were performed connecting a digital flow meter before and after the ML-800 reference standard.

The results appear in Figure 3.

The difference between the reference and UUT values are smaller when the UUT is connected in line with the reference standard than to the outlet of it, except for the $0.1 \mathrm{~L} / \mathrm{min}$ flow rate. The measurement results show that the biggest differences in the calibration position were measured at the middle of the calibration range. At a nominal flow rate of $3 \mathrm{~L} / \mathrm{min}$ the difference is $0.044 \mathrm{~L} / \mathrm{min}$ and at $4 \mathrm{~L} / \mathrm{min}$ the difference is $0.049 \mathrm{~L} / \mathrm{min}$. The smallest difference occurs at $0.1 \mathrm{~L} / \mathrm{min}$ and $1 \mathrm{~L} / \mathrm{min}$. At the nominal flow rates of $2 \mathrm{~L} / \mathrm{min} ; 3 \mathrm{~L} / \mathrm{min} ; 4 \mathrm{~L} / \mathrm{min}$ and $6 \mathrm{~L} / \mathrm{min}$, measurement uncertainties are also not overlapping. The differences between the reference and UUT values for the two calibration positions at the above mentioned flow rates are bigger than the uncertainty values 


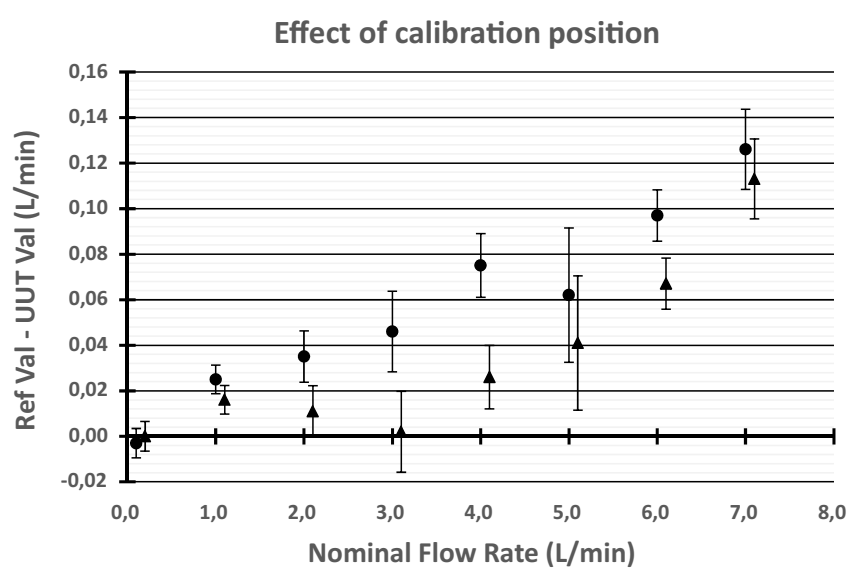

- Connected to the outlet

$\Delta$ Connected in line

Fig. 3. Flow measurement values illustrating the effect of the calibration position of the UUT.

at these points. The uncertainty value at $5 \mathrm{~L} / \mathrm{min}$ is relatively larger than the other uncertainties which resulted from poor repeatability at this point.

The two calibration methods indicate a measurable difference in results.

\subsection{Effects of gas supply pressure}

Currently the calibration method used in the laboratory requires that a gas supply pressure of $200 \mathrm{kPa}$ be used. In order to establish whether or not the gas supply pressure influences the measurement results, measurements were performed in which a digital flow meter was calibrated at a nominal flow rate of $7 \mathrm{~L} / \mathrm{min}$ against the ML- 800 and the supply gas pressure varied between $50 \mathrm{kPa}$ and $300 \mathrm{kPa}$.

The results can be seen in Figure 4.

The worst case difference between the flow rate values of the reference standard and the UUT in the pressure range $50 \mathrm{kPa}$ to $300 \mathrm{kPa}$, was $0.023 \mathrm{~L} / \mathrm{min}$. Due to the fact that this difference falls within overlapping uncertainties, the effect of the gas supply pressure between $50 \mathrm{kPa}$ and $300 \mathrm{kPa}$ can be considered insignificant.

\section{Calculation of measurement uncertainties}

Uncertainties are calculated according to the Guide to the Expression of Uncertainty in Measurement.

The following sources of uncertainty are considered with each calibration:

\section{a) ML-800 accuracy}

Since the calibration certificates for the Flow Cells provided evidence that their accuracy was well within the manufacturer's accuracy specification, the manufacturer's accuracy specification was accepted as the uncertainty estimate. It was treated as a normal distribution variable, at $k=2$ and therefore divided by two.

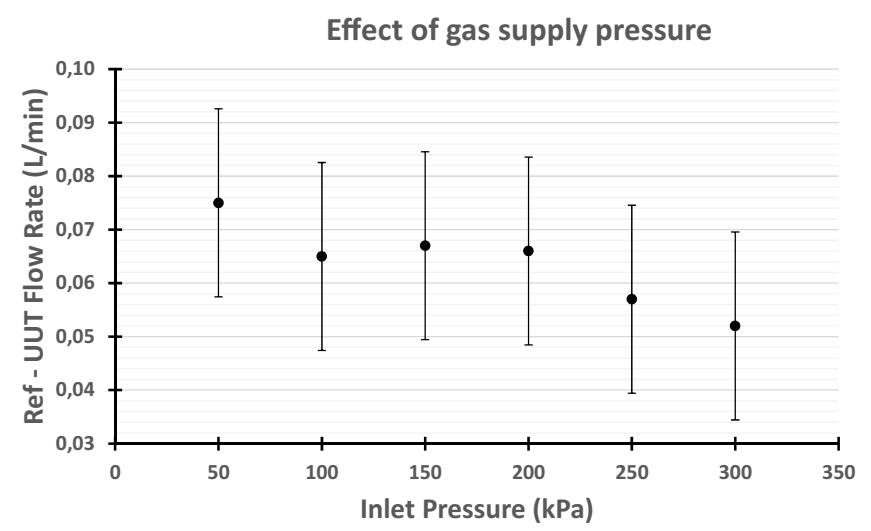

Fig. 4. Flow measurement values indicating the effect of gas supply pressure.

\section{b) Temperature}

No uncertainty contribution for temperature is considered since the ML-800 sensitivity to temperature is unknown. However, since the ML- 800 is used in a laboratory environment within that specified by the manufacturer for the ML-800 to remain within its accuracy specification, the effect of temperature is assumed to be negligible.

c) Pressure

No uncertainty contribution for pressure is considered since the ML-800 sensitivity to pressure was unknown. The ML-800 compensated for atmospheric pressure, as measured by its internal barometer.

d) Repeatability of measurements (ESDM) - standard and unit under test as a combined system

The experimental standard deviation of the mean is used as the uncertainty estimate for the variation of repeated measurements, since the measurements are performed under repeatable conditions (conditions remained the same) and the calculated mean value of ten independent flow measurements is reported. The degrees of freedom are the number of measurements minus one, which for ten measurements is nine.

\section{e) Resolution of ML-800}

The displayed resolution of the ML-800 is dependent on which flow rate is being measured. Therefore the uncertainty estimate is accepted as one least significant digit of the displayed flow. Since this is the full range of a rectangular distributed input variable, it is divided by two and then further divided by the square root of three. It is assigned infinite degrees of freedom.

f) Resolution of the unit under test

The uncertainty estimate is accepted as one least significant digit of the displayed flow. Since this is the full range of a rectangular distributed input variable, it is divided by two and then further divided by the square root of three. It is assigned infinite degrees of freedom. 
Uncertainty estimates are calculated in absolute values. Measurement uncertainties are calculated for each measurement point. Uncertainty calculations indicate the most significant contributors to be the UUT display resolution and system repeatability [1].

\section{Conclusions and recommendations}

The validation described in Section 4 was unsatisfactory in achieving the method target measurement uncertainty. However, the results provided confidence that no damage had been sustained during shipment of the ML-800 from the USA.

A bilateral comparison has therefore been planned with an overseas National Metrology Institute to validate the calibration method adequately. Mass flow controllers with a dedicated read-out and control unit, with a short calibration interval will be used as transfer standards.

Initial measurements to determine the effects of UUT calibration position (Sect. 5.1) indicate possible significant effects.
Initial measurements to determine the effects of gas supply pressure (Sect. 5.2) indicate possible insignificant effects.

However, due to the fact that only one instrument was used for these measurements, the sample was not large enough to represent all instruments. It is therefore recommended that further measurents be performed on a wider range of instrumentation.

\section{References}

1. D. Jonker, E.P. Tarnow, NMISA new gas flow calibration facility, Proceedings of Test $\&$ Measurement 2013 Conference E Workshop (2013)

2. Bios Met Lab Series ML-800 User Manual, 2011, Bios International Corporation, MK-01-33 Rev. J.

3. D. Jonker, NMISA-12-0218, Validation report of the Bios Met Lab ML-800 primary flow standard, 2013 